\title{
Private general paediatric care availability in Melbourne
}

\author{
Marina Kunin ${ }^{1} \mathrm{MA}, \mathrm{PhD}$, Research Fellow \\ Amy R. Allen ${ }^{1}$ BBNSc, BPsySci (Hons), GradDipAOD, Research Assistant \\ Caroline Nicolas ${ }^{1}$ BSocSc(Psych), Research Coordinator \\ Gary L. Freed ${ }^{1,2} \mathrm{MD}, \mathrm{MPH}$, Director \\ ${ }^{1}$ Centre for Health Policy, Melbourne School of Population and Global Health, The University of Melbourne, \\ 207 Bouverie Street, Carlton, Vic. 3053, Australia. Email: marina.kunin@unimelb.edu.au; \\ amy.allen@unimelb.edu.au; cnicolas@live.com.au \\ ${ }^{2}$ Corresponding author. Email: gary.freed@unimelb.edu.au
}

\begin{abstract}
Objective. The aims of the present study were to determine the actual availability of private general paediatric appointments in the Melbourne metropolitan region for children with non-urgent chronic illnesses and the cost of such care.

Methods. A 'secret shopper' method was used. Telephone calls were made to a random sample of 47 private paediatric clinics. A trained research assistant posed as a parent, requesting the first available appointment with a specific paediatrician. Data regarding appointment availability, total potential charges and net charges after the Medicare rebate were collected.

Results. Appointments were available in 79\% $(n=37)$ of clinics, with $72 \%(n=34)$ able to offer an appointment with the requested general paediatrician. The number of days until available appointments varied from same day appointments to a wait of 124 days, with an average wait of 33 days. Of practices that provided information about the appointment cost $(n=42)$, five bulk-billed for the consultation, whereas the remainder $(n=37)$ were fee-paying clinics. The potential maximum charge for an initial consultation in the fee-paying clinics ranged from $A \$ 177$ to A $\$ 430$, with an average cost of $A \$ 279$. The potential maximum out-of-pocket cost for patients ranged from $A \$ 40$ to A\$222, with an average outof-pocket cost of A\$128.

Conclusions. Private paediatric care in the Melbourne metropolitan region is generally available. The out-of-pocket cost of private paediatric out-patient care may present a potential economic barrier for some families.

What is known about the topic? In Australia, out-of-pocket expenses for private specialist care are not covered by private health insurance. There are no data available on the actual cost of private paediatric consultations that are based on real-time assessments. Data collected in 1998 suggested that the average waiting time for a first standard consultation with a general paediatrician in a private room was 14.1 days. There are no recent empirical data on appointment availability and waiting time for appointments with general paediatricians in Australia.

What does this paper add? There is high availability of paediatric consultations in the private sector. Waiting times for an appointment vary considerably from same day appointments to a wait of 124 days, with an average wait of 33 days. The cost of a private paediatric consultation in Australia to the patient is considerable, with an average potential maximum up-front charge for an initial consultation of A \$279 and an average potential maximum out-of-pocket cost of A\$128.

What are the implications for practitioners? Data on the availability and cost of private paediatric consultations are imperative to formulate evidence-informed policy and better understand variations in the availability of public and private care.
\end{abstract}

Received 13 November 2015, accepted 9 February 2016, published online 24 March 2016

\section{Introduction}

In Australia, ambulatory general paediatric care is provided by general paediatricians, who are specialist practitioners working in either private or public settings. The public-private mix of service delivery and financing is common throughout the Australian health system; however, concerns exist that access to health services is becoming less equitable. ${ }^{1}$ To understand potential barriers to ambulatory paediatric care, it is important to assess appointment availability and any potential financial burden that may impede access to general paediatricians.

In the public sector, patients can receive care from a general paediatrician free of charge in out-patient clinics of public 
hospitals that are funded through Medicare or from the state budget. Community health services, funded by the states, also provide free paediatric care. ${ }^{2}$ However, some patients may elect to pursue general paediatric care privately. In the private sector, general paediatricians charge a fee for service and either 'bulkbill' Medicare (i.e. accept the Medicare benefit as full payment for the services rendered) or bill patients directly. Medicare benefits are based on the Medicare Benefits Schedule (MBS), a listing of the fees for the Medicare services paid by the Commonwealth Government. A paediatrician may charge a higher amount than a Medicare schedule fee. In these instances, patients may claim the Medicare benefit amount back from Medicare ( $85 \%$ of the schedule fee for a specialist consultation), termed the Medicare rebate, but will pay out-of-pocket expenses for the gap between the consultation fee and the rebate. ${ }^{2}$

Out-of-pocket expenses for specialist care are not covered by private health insurance in Australia (Private Health Insurance Act 2007 No. 31, 2007, Australia) and may present a substantial financial burden to patients. Nationally, expenditure on specialist doctors' fees represents $10 \%$ of Australian households' weekly expenditure on health. ${ }^{3}$ The average charge for a private paediatric consultation for a newborn (with no complications) has been estimated to be A $\$ 250-400$, resulting in an out-of-pocket expense of A\$125-275 after the Medicare rebate. ${ }^{4}$ It is difficult to determine the extent to which the cost of general paediatric consultations may act as a barrier to access, because there are no data available on the actual cost of private paediatric consultations that are based on real-time assessments.

In addition, there are no recent empirical data on appointment availability and waiting times for appointments with general paediatricians in Australia. Data collected in 1998 suggested that the average waiting time for a first standard consultation with a general paediatrician in a private room was 14.1 days, whereas patients being seen in public hospital-based out-patient clinics waited, on average, 36 days. ${ }^{5}$ These findings have not been updated for more than 15 years and are based on self-reports from Australian Medical Workforce Advisory Committee (AMWAC) surveys, not actual availability and accessibility of such services. Data regarding wait times for an appointment in other paediatric services suggest much longer periods: 5 months for paediatric weight management services ${ }^{6}$ and from 5.4 (private) to 10.6 months (public) for paediatric allergy services. ${ }^{7}$ These reports too were based on the estimates of service providers, not actual availability of the service. No previous studies to date have provided a real-time assessment of general paediatricians availability from a patient perspective.

The aims of the present study were to determine the actual availability and charges for general paediatric services in the private sector for children with chronic conditions of low acuity and urgency. The study used actual, real-time assessments from a patient perspective.

\section{Methods}

Study design

The study used an audit methodology with 'secret shopper' techniques, a well-recognised method in healthcare research, to investigate the actual availability of services. ${ }^{8-10}$

\section{Sample}

The sampling frame consisted of general paediatricians working in paediatric practices in the Melbourne metropolitan region. A list of general paediatricians was derived from the Human Services Directory website (http://humanservicesdirectory.vic. gov.au/Home.aspx, accessed June 2014). A sample of 47 general paediatricians was randomly selected using a computer-generated random number sequence. Paediatricians were excluded from the sample if they were subspecialists (e.g. paediatric cardiologist, paediatric neurologist) or were in practices where a telephone call had already been made to another practitioner as part of the study.

\section{Data collection}

Data were collected from August to October 2014. One of two scripted clinical scenarios was used by a research assistant who telephoned to the general paediatrician practices posing as a parent. Both scenarios were tailored to represent non-urgent chronic conditions, commonly treated within a general paediatric setting: (1) a 3-year-old boy with asthma, referred by a general practitioner (GP); and (2) an 8-month-old girl with eczema, referred by a GP. The scenarios were developed with the assistance of an advisory group consisting of two clerical staff from two paediatric practices and two general paediatricians in practice.

A single telephone call was made to each practice. A research assistant asked for the first available appointment with a specific paediatrician. If this was not available, the research assistant asked for the first available appointment with any paediatrician within the practice. Data were collected on the following:

- availability of an appointment with the requested or alternative paediatrician

- wait time (number of days) until the appointment

- reasons for the lack of an available appointment

- total charge to parents of an initial appointment (if told that the charge for an appointment would vary depending on the length and level of complexity of the consultation, a minimum and maximum charge provided by the practice was recorded).

Where an appointment time was offered, the research assistant deferred from actually accepting the appointment and stated they would call back if an appointment was required. No appointments were actually booked in any general paediatric practices. The call process was developed and pilot tested with medical reception staff from paediatric clinics to ensure it did not create a significant burden on the clinics.

\section{Data analysis}

Descriptive statistics were used to explore the availability and cost of appointments. The analysis was conducted using statistical software package Stata Statistical Software Release 13 (StataCorp LP, College Station, TX, USA).

The University of Melbourne Human Research Ethics Committee approved this study and required debriefing letters to be sent to the practices informing them of the nature of the study and that they had been contacted by the research team. 


\section{Results}

In all, 47 general paediatricians were contacted, 18 from the northern and north-western suburbs of Melbourne and 29 from the eastern and south-eastern suburbs.

\section{Appointment availability}

Appointments were offered in 79\% $(n=37)$ of clinics contacted, with $72 \%(n=34)$ being available with the requested general paediatrician. The most frequent reason for an appointment not being offered was the requirement for a referral letter from a GP to be received before securing an appointment (Table 1).

The number of days until available appointments varied substantially from same day appointments to a wait of 124 days, with an average wait of 33 days. More than one-third of practices with an available appointment $(38 \% ; n=14)$ had waiting periods ranging from 42 to 124 days. However, a similar percentage of practices where the appointment was available were able to offer an appointment within 1 week $(35 \% ; n=13$; Table 2$)$.

\section{Appointment cost}

Most practices $(89 \% ; n=42)$ provided information about the appointment cost. Of these, five would bulk-bill for the consultation, whereas others $(88 \% ; n=37)$ quoted charges above the Medicare rebate requiring an out-of-pocket payment ('fee-paying practices').

The minimum and maximum up-front charges quoted for the initial consultation in fee-paying practices ranged from A \$177 to A \$430. The average potential maximum charge for an initial consultation was A $\$ 279$. After application of the Medicare rebate, out-of-pocket costs for patients would range

Table 1. Paediatric practices, by appointment availability Data are given as $n(\%)$

\begin{tabular}{lc}
\hline Appointment availability & \\
\hline Appointment offered with the requested paediatrician & $34(72 \%)$ \\
Appointment offered with another paediatrician & $3(6 \%)$ \\
No appointment offered & $10(21 \%)$ \\
Reasons for no appointment being offered & \\
Referral letter needs to be sent first & $5(11 \%)$ \\
Not taking new patients & $2(4 \%)$ \\
No available appointments & $1(2 \%)$ \\
Paediatrician on leave & $1(2 \%)$ \\
Other & $1(2 \%)$ \\
Total & $47(100 \%)$ \\
\hline
\end{tabular}

Table 2. Days to the appointment Paediatric practices with available appointment, $n=37$

\begin{tabular}{lcc}
\hline & No. practices (\%) & $\begin{array}{c}\text { Mean time to } \\
\text { appointment (days) }\end{array}$ \\
\hline 1 week (0-7 day) & $13(35)$ & 4 \\
2 weeks (8-14 days) & $2(5)$ & 11 \\
3 weeks (15-21 days) & $3(8)$ & 19 \\
4 weeks (22-28 days) & $5(14)$ & 22 \\
$>4$ weeks & $14(38)$ & 69 \\
Total (0-124 days) & $37(100)$ & 33 \\
\hline
\end{tabular}

from $\mathrm{A} \$ 40$ to $\mathrm{A} \$ 222$, with an average potential maximum cost of A $\$ 128$ (Table 3). For $38 \%$ of consultations $(n=16)$, the maximum potential out-of-pocket cost for the initial consultation exceeded A\$150 (Table 4).

\section{Discussion}

Among the most important findings in the present study is the high availability of paediatric consultations in the private sector. However, waiting times for an appointment varied considerably across the sample and, importantly, there was an out-of-pocket cost for most of these consultations.

The fact that only three practices declined to book an appointment, either because they were not accepting new patients or had no appointments available, indicates that most families seeking a private paediatric consultation would be successful. However, depending on family financial circumstances and parent perceived urgency of care, barriers to accessibility may exist.

The average wait time for an appointment among the sample of general paediatricians in the present study was 33 days. This is more than twice as long as the 1998 results from the only other published data of this kind. ${ }^{5}$ The rise in waiting times for an appointment may represent an increase in demand for paediatric consultations in the private sector over the past 15 years. This increase may be due to the rise in the absolute number of children in Australia (by 6\%) during this period. ${ }^{11}$ It is possible that this increase is tied to the change in the volume and patterns of paediatric care among GPs in Australia. During the corresponding period, paediatric consultations became a smaller fraction of overall GP visits, ${ }^{12}$ whereas the absolute number and proportion of longer consultations for children have decreased. ${ }^{13}$ The observed increase in waiting times for a paediatric consultation in the present compared with the previous study ${ }^{5}$ may also be a result of our use of the audit methodology ('secret shopper'), which recorded the actual availability of the service at a given time, rather than physician self-reported recall.

Table 3. Appointment costs (A\$)

\begin{tabular}{lcccc}
\hline & \multicolumn{2}{c}{ Up-front charges } & \multicolumn{2}{c}{ Out-of-pocket charges } \\
& Minimum & Maximum & Minimum & Maximum \\
\hline Range & $177-430$ & $177-430$ & $40-222$ & $60-222$ \\
Mean & 269 & 279 & 124 & 128 \\
\hline
\end{tabular}

Table 4. Consultation costs, according to minimum and maximum out-of-pocket charges

Data show the number of practices in each group, with percentages in parentheses

\begin{tabular}{lrr}
\hline & \multicolumn{2}{c}{ Out-of-pocket charges } \\
& Minimum & \\
\hline Consultation charges (A\$) & & $5(12 \%)$ \\
Bulk-billing & $5(12 \%)$ & $8(19 \%)$ \\
$<80$ & $8(19 \%)$ & $13(31 \%)$ \\
$80-149$ & $17(41 \%)$ & $16(38 \%)$ \\
$\geq 150$ & $12(29 \%)$ & \\
\hline
\end{tabular}


Because of the design of the present study, we are unable to explain the high variability of waiting times across the clinics (from same day appointments to a wait of 124 days). Additional research is needed to explore whether this variability signifies a mismatch between demand and supply for paediatrician services across different areas of Melbourne. Studies are also needed to assess whether there is a correlation between the wait time for an appointment and the consultation cost or whether this phenomenon can be linked to parent preferences for a specific paediatrician.

The results showed that the cost of a private paediatric consultation in Australia to the patient is significant, with an average maximum up-front charge of $\mathrm{A} \$ 279$. Because there is no system for private insurance cover for out-patient specialist visits, patients are responsible for all charges above the Medicare rebate. We found the average potential maximum out-of-pocket cost for an initial visit was $\mathrm{A} \$ 128$.

Previous research has shown that high user fees and out-ofpocket payments have a negative impact on health equity, putting a heavier burden on sick and poor members of society. ${ }^{14,15}$ Consultation costs can have a significant effect on patients' ability to access private healthcare. A survey of patient experiences in Australia revealed that of those needing to see a medical specialist in 2013-14, 8\% delayed or did not access the service due to cost. ${ }^{16}$ A study among older Australians found an association between the number of chronic medical conditions and inability to afford out-of-pocket expenses. ${ }^{17}$ No such study exists for paediatric care. In Australia, these expenses commonly include pharmaceuticals, medical aids and appliances and medical services (i.e. out-of-pocket payments to doctors who do not bulk bill services and other healthcare professionals). ${ }^{2}$

A separate study has shown that out-of-pocket expenditure for healthcare per capita in Australia is higher than the average for Organization For Economic Cooperation and Development (OECD) countries. ${ }^{18}$ Per capita, out-of-pocket expenditure in Australia increased more than $70 \%$ since 2000 , from US\$452 purchasing power parity (PPP) to US\$771 PPP in 2012. ${ }^{19}$ Globally, people living in countries with higher out-of-pocket payments were found to face catastrophic healthcare expenditure more frequently compared with their counterparts living in countries where a larger part of healthcare system is financed through health insurance or other prepaid health financing mechanisms. $^{20}$

One of the reasons for the increase in out-of-pocket expenses for medical consultations in Australia has been attributed to the freeze of Medicare rebates. ${ }^{21}$ The government has not updated the Medicare rebates for GP and specialist consultations in accordance with the consumer price index (CPI) since 1 November 2012. In the 2014-15 federal budget, this freeze was extended until 1 July $2018 .^{21}$ A study that examined the effect of this freeze on GP income found that by 2017-18, based on CPI rises, GPs would be losing $7.1 \%$ of the rebate income compared with 2014-15, thus needing to increase their consultation fees to recoup income lost through the freeze. ${ }^{22}$ For patients, this would result in a higher out-of-pocket pay for the gap between the consultation fee and the Medicare rebate. There is likely to be similar upward pressure on paediatrician consultation fees.

In Australia, patients can receive free care from a general paediatrician in the public sector in one of the paediatric out-patient clinics of the public hospitals or at a maternal infant clinic. However, the availability of these services is more limited, with waiting times for appointments in the public sector being roughly twice as long as in the private sector. ${ }^{5,7}$ In addition, recently published data on waiting times in some public outpatient adult specialist clinics showed that the wait for an appointment varies considerably across Victorian hospitals. For example, in 2015, median wait for a first appointment at a general medicine clinic in the Bendigo Health Care Group was 91 days, whereas the median wait for the same type of specialist in Melbourne Health was 15 days. $^{23}$

There are some limitations to the present study. The small sample size only allows descriptive analysis of our data. The data were collected during winter-spring 2014 and seasonal issues (more viral illnesses in winter) could affect consultation availability. In addition, the sample was taken only from the Melbourne metropolitan area and, as such, these data may not be generalisable to other areas of Australia. Further research is required to assess paediatrician availability and out-of-pocket costs in other areas of the country. However, the main strength of the present study lies in the use of a 'secret shopper' method that enabled the collection of real-time data on availability and cost of paediatric consultations in the private sector from the patient perspective.

\section{Conclusion}

Waiting times for private paediatric consultations in the Melbourne metropolitan area are highly variable. The out-ofpocket cost of private paediatric out-patient care may present a potential economic barrier for some families. Data on the availability and cost of private paediatric consultations are imperative to formulate evidence-informed policy and better understand the variation in public and private care availability.

\section{Competing interests}

None declared.

\section{Acknowledgements}

The authors thank the participants for their time answering the survey. This study was funded by the Victorian Government's Department of Health and Royal Children's Hospital Foundation.

\section{References}

1 Armstrong BK, Gillespie JA, Leeder SR, Rubin GL, Russell LM. Challenges in health and health care for Australia. Med J Aust 2007; 187: 485-9.

2 Healy J, Sharman E, Lokuge B. Australia: health system review. Health Syst Transit 2006; 8: 1-158.

3 Australian Bureau of Statistics. Year book Australia, 2012. Canberra: ABS; 2012.

4 Nib Health Funds Limited. The costs of having a baby. Private system. Nib health funds; NSW. Available at: https://www.nib.com.au/healthinformation/content/assets/pdf/nib-costs-of-baby-guide.pdf [verified August 2015].

5 Australian Medical Workforce Advisory Committee. The consultant paediatric workforce in Australia. Report 1999.6 Sydney: AMWAC; 1999. Available at: http://www.ahwo.gov.au/documents/Publications/ 1999/The\%20consultant\%20paediatric\%20workforce\%20in\%20Australia. pdf [verified August 2015]. 
6 Spilchak PJ, Denney-Wilson E, King L, Baur LA. Tertiary paediatric obesity services in Australia. J Paediatr Child Health 2008; 44: 243-7. doi:10.1111/j.1440-1754.2007.01266.x

7 Morawetz DY, Hiscock H, Allen KJ, Davies S, Danchin MH. Management of food allergy: a survey of Australian paediatricians. $J$ Paediatr Child Health 2014; 50: 432-7. doi:10.1111/jpc.12498

8 Chaudhry SB, Armbrecht ES, Shin Y, Matula S, Caffrey C, Varade R, Jones L, Siegried E. Pediatric access to dermatologists: Medicaid versus private insurance. J Am Acad Dermatol 2013; 68: 738-48. doi:10.1016/ j.jaad.2012.10.034

9 Bisgaier J, Rhodes KV. Auditing access to specialty care for children with public insurance. $N$ Engl J Med 2011; 364: 2324-33. doi:10.1056/ NEJMsa1013285

10 Freed GL, Bingham A, Allen AR, Freed M, Sanci LA, Spike N. Actual availability of general practice appointments for mildly ill children. Med J Aust 2015; 203: 145-145e.5. doi:10.5694/mja14.01724

11 Australian Bureau of Statistics. Australian demographic statistics, table 59. Estimated resident population by single year of age, Australia. Canberra: ABS; 2014.

12 Freed GL, Sewell J, Spike N, Moran L, Brooks P. Changes in the demography of Australia and therefore general practice patient populations. Aust Fam Physician 2012; 41: 715-19.

13 Freed GL, Spike NA, Sewell JR, Moran LM, Britt H, Valenti L, Brooks P. Changes in longer consultations for children in general practice. J Paediatr Child Health 2013; 49: 325-9. doi:10.1111/jpc.12157

14 Burström B. Increasing inequalities in health care utilisation across income groups in Sweden during the 1990s? Health Policy 2002; 62: 117-29. doi:10.1016/S0168-8510(02)00016-7

15 Duckett S, Breadon P. Out-of-pocket costs: hitting the most vulnerable hardest. Grattan Institute submission to the Senate Standing Committee on Community Affairs Inquiry into the out-of-pocket costs in Australian healthcare. Melbourne: Grattan Institute; 2014.
16 Australian Bureau of Statistics. Patient experiences in Australia: summary of findings, 2013-14. Canberra: ABS; 2015.

17 Carpenter A, Islam MM, Yen L, McRae I. Affordability of out-of-pocket health care expenses among older Australians. Health Policy 2015; 119 : 907-14. doi:10.1016/j.healthpol.2015.03.010

18 Organization For Economic Cooperation and Development (OECD). OECD health statistics 2014 - frequently requested data. 2014. Available at: http://www.oecd.org/els/health-systems/oecd-health-statistics-2014frequently-requested-data.htm [verified 15 August 2015].

19 World Health Organization (WHO). Primary health care - now more than ever. Geneva: WHO; 2008

20 Rollins A. 'Lazy' rebate freeze should join co-payment on scrapheap: AMA. Aust Med 2015. Available at: https://ama.com.au/ausmed/\%E2\% $80 \% 981$ lazy $\%$ E2 $\% 80 \% 99$-rebate-freeze-should-join-co-payment-scrapheap-ama [verified 15 August 2015].

21 Australian Government. Budget 2014-15: budget measures. Canberra: Commonwealth of Australia; 2014. Available at: http://budget.gov.au/ 2014-15/content/bp2/download/BP2_consolidated.pdf [verified October 2015].

22 Harrison C, Bayram C, Miller GC, Britt HC. The cost of freezing general practice. Med J Aust 2015; 202: 313-16. doi:10.5694/mja15. 00182

23 System Intelligence and Analytics Branch, Department of Health and Human Services. Specialist clinics activity and wait time report, June quarter 2014-15. Melbourne: Victorian Government; 2015. Available at: file://CC:/Users/mkunin/Downloads/Specialist\%20Clinics\%20Quarterly \%20Activity\%20and\%20Wait\%20Time\%20Report\%20June\%202015\% 20Quarter\%20-\%20Final.pdf [verified October 2015]. 